level indicators of injury morbidity and mortality involves systemic change.

Description of problem We will not solve the public health problem of injury simply by educating individuals about the nature of injury risk, improving their risk assessment, and providing these individuals with information to enable them to reduce the level of risk to which they are exposed. Substantial improvement in the societal injury burden will occur only when changes are made at the societal level that focus on reducing the population level indicators of injury related harm.

Results In this presentation, we consider a shift from the contemporary systematic approach to injury and violence prevention, to a systemic approach more consistent with the principles of ecological public health. We consider the extent to which the logic of the systematic model, and the related misconceptions about the role of uncertainty in science, limit local, national, and global efforts to minimise injury related harm. The shift from an individual to a population perspective has substantial implications for the way we perceive, direct, undertake, and evaluate injury prevention research and practice. The analogy of "the population as patient" provides a clear illustration of the foundational truths that underpin the preferred public health approach to the prevention of injury.

Conclusions We conclude by delineating a new program of work that could be of considerable benefit to the injury-related health of populations.

\section{PROMOTING SOCIAL AND COMMUNITY CHANGES AS THE BASIS FOR SUICIDE PREVENTION}

Eric D Caine. Injury Control Research Centre for Suicide Prevention, and Department of Psychiatry University of Rochester Medical Centre Rochester, NY 14642 USA

10.1136/injuryprev-2016-042156.37

To date, there have been no effective, broadly applied, comprehensive and sustainable approaches to preventing suicide and risk-related premature deaths in the United States. Rare examples of exceptional programs exist - in the US Air Force and the police force of Montreal, Quebec, Canada - but no one has replicated these results, nor is it clear that they are suitable for dealing with the extraordinary diversity within and between states, let alone an entire country. Preventing suicides and premature deaths - to the extent that there is a substantial reduction in populationlevel rates - will require systemic, systematically applied and coordinated interventions. They will require carefully crafted public health initiatives that reach far 'upstream' while also dealing with persons on the 'edge of death.' While suicide is often viewed from the perspectives of individuals who have killed themselves, these deaths are drawn from diverse groups who share common characteristics. Effective prevention programs must address the diversity of these groups even as it is essential to meet the needs of individuals suffering great distress. Suicide prevention must be built as a mosaic; no single piece will convey the entire picture.

This presentation will consider an approach developed with a partner State to design and implement a "full court press" approach necessary for creating and sustaining the mosaic of efforts required for preventing suicide, attempted suicide, and antecedent risks that are common to these adverse outcomes, as well as other related forms of premature death. It will consider the challenges involved when promoting effective, broad based coalitions; the opportunities from forging synergies among diverse groups; the development of dynamic models to explore the impact of programmatic changes and interventions; and the central role of "culture change" that will be essential to creating and sustaining a powerfully effective suicide prevention movement.

\section{INJURY PREVENTION AS A BYPRODUCT}

Ronan A Lyons. Swansea University Medical School

\subsection{6/injuryprev-2016-042156.38}

Background It is plausible to suggest that population changes in injury incidence and outcomes require sustained changes in societal level determinants. However, social change takes time and effectiveness of the approach is difficult to demonstrate using standard research paradigms.

Description of problem To our knowledge there are no convincing reports in the scientific literature of successful, coordinated efforts to develop and implement a systems-level injury prevention interventions based on an initial elucidation of societal level determinants of that population's level injury rates. In this presentation we describe work we conducted over many years in relation to a wide variety of injuries types, and an array of legal, policy and environmental interventions. Using empirical examples to illustrate our points, we aim in this presentation to i) develop a picture of what a systems level intervention might look like, ii) explain what "implementation" means in this context and iii) discuss the challenges and potential benefits of a societal approach.

Results The presented examples demonstrate three findings. First, it is extremely difficult to encourage stakeholder committment to building interventions in the community "from the ground up"; Targetted solutions to circumscribed, technical problems, are almost universally preferred. Second, targeted interventions, apparently successfull in terms of specific outcomes, may not in fact be successfull if all relevant outcomes are included in the effectiveness analyses. Third, for many types of injury we will achieve greater successful by not explicitly attempting to reduce injury risk (most people believe that injuries will not happen to them) but to engage the population in activities they value, such as increasing resilience and independence, and that produce fewer injuries as a byproduct.

Conclusions While the systemic approach to injury prevention has face validity as a concept. There is insufficient evidence of its effectiveness as a prescription for prevention practice.

\section{Pre-Conference Sessions Sunday 18.9.2016 13:00-14:45}

\section{SU IP W2}

\section{CHILD AND ADOLESCENT INJURIES IN THE ARAB GULF: UNDERSTANDING THE BURDEN AND RISK FACTORS}

${ }^{1}$ Adnan A Hyder, ${ }^{1}$ Amber Mehmood, ${ }^{2}$ Ruben Peralta, ${ }^{2}$ Rafael Consunji, ${ }^{1}$ Kate Allen. ${ }^{1} J o h n s$ Hopkins International Injury Research Unit, USA; ${ }^{2}$ Hamad Medical Corporation, Qatar

10.1136/injuryprev-2016-042156.39

Background The WHO estimates that every day 2,000 children and adolescents are killed by preventable injuries. In the high- 